\title{
Nuclear E-cadherin and VHL immunoreactivity are prognostic indicators of clear-cell renal cell carcinoma
}

\author{
Michelle L Gervais ${ }^{1}$, Pauline C Henry ${ }^{2}$, Arthy Saravanan ${ }^{2}$, T Nadine Burry ${ }^{1}$, Brenda L Gallie ${ }^{3}$, Michael AS Jewett ${ }^{4}$ \\ Richard P Hill ${ }^{5}$, Andrew J Evans ${ }^{2}$ and Michael Ohh ${ }^{1}$
}

The loss of functional von Hippel-Lindau (VHL) tumor suppressor gene is associated with the development of clear-cell renal cell carcinoma (CC-RCC). Recently, VHL was shown to promote the transcription of E-cadherin, an adhesion molecule whose expression is inversely correlated with the aggressive phenotype of numerous epithelial cancers. Here, we performed immunohistochemistry on CC-RCC tissue microarrays to determine the prognostic value of E-cadherin and VHL with respect to Fuhrman grade and clinical prognosis. Low Fuhrman grade and good prognosis associated with positive VHL and E-cadherin immunoreactivity, whereas poor prognosis and high-grade tumors associated with a lack of E-cadherin and lower frequency of VHL staining. A significant portion of CC-RCC with positive VHL immunostaining correlated with nuclear localization of C-terminally cleaved E-cadherin. DNA sequencing revealed in a majority of nuclear E-cadherin-positive CC-RCC, subtle point mutations, deletions and insertions in VHL. Furthermore, nuclear E-cadherin was not observed in chromophobe or papillary RCC, as well as matched normal kidney tissue. In addition, nuclear E-cadherin localization was recapitulated in CC-RCC xenografts devoid of functional VHL or reconstituted with synthetic mutant VHL grown in SCID mice. These findings provide the first evidence of aberrant nuclear localization of E-cadherin in CC-RCC harboring VHL mutations, and suggest potential prognostic value of VHL and E-cadherin in CC-RCC.

Laboratory Investigation (2007) 87, 1252-1264; doi:10.1038/labinvest.3700684; published online 1 October 2007

KEYWORDS: CC-RCC; nuclear E-cadherin; prognostic indicators; tissue microarray; VHL

Renal cell carcinoma (RCC) accounts for approximately $3 \%$ of all adult malignancies, with the clear-cell type (CC-RCC) comprising $80 \%$ of RCC. ${ }^{1,2}$ Approximately $25-30 \%$ of CC-RCC patients have metastatic disease at diagnosis, and $20-30 \%$ of patients with clinically localized CC-RCC develop metastasis post-nephrectomy. ${ }^{3}$ Recent advances in the use of antiangiogenesis-targeted agents appear to have revolutionized treatment. ${ }^{4-7}$ Currently, a prediction of patient survival is based on traditional clinical parameters, including tumor size and Fuhrman nuclear grade. ${ }^{8}$ However, the emerging understanding of the molecular pathways implicated in CC-RCC genesis and progression is providing previously unappreciated markers, which may serve as additional or better prognostic indicators of CC-RCC.

The principal cause of sporadic CC-RCC and familial von Hippel-Lindau (VHL) disease-associated CC-RCC is the inactivating mutations of VHL. Although VHL patients also develop tumors in other organs including the central nervous system, retina and the adrenal gland, CC-RCC remains to be the leading cause of morbidity and death for VHL patients. ${ }^{9}$ The most well-established function of VHL is its role in the oxygen-dependent negative regulation of hypoxia-inducible factor (HIF). VHL is a substrate-conferring component of an E3 ubiquitin ligase ECV (elongins/Cul2/VHL) that polyubiquitylates the catalytic $\alpha$-subunit of HIF that has undergone hydroxylation on conserved prolyl residues within the oxygen-dependent degradation (ODD) domain. Prolyl hydroxylation is mediated by a class of prolyl hydroxylases (PHD1-3) in an oxygen-dependent manner. Thus, under hypoxia or in the absence of a functional VHL, HIF $\alpha$ becomes stabilized and binds to its common and constitutively expressed $\beta$-subunit, forming an active HIF transcription factor capable of transactivating numerous hypoxia-inducible genes such as vascular endothelial growth factor (VEGF),

\footnotetext{
${ }^{1}$ Department of Laboratory Medicine and Pathobiology, Faculty of Medicine, University of Toronto, Toronto, ON, Canada; ${ }^{2}$ Department of Pathology, University Health Network, Toronto, ON, Canada; ${ }^{3}$ Division of Cancer Informatics, Ontario Cancer Institute/Princess Margaret Hospital, University Health Network, Toronto, ON, Canada; ${ }^{4}$ Departments of Surgery/Urology, University Health Network, Princess Margaret Hospital, University of Toronto and Surgical Oncology, Toronto, ON, Canada and ${ }^{5}$ Department of Medical Biophysics, Ontario Cancer Institute, Princess Margaret Hospital, University of Toronto, Toronto, ON, Canada Correspondence: Dr M Ohh, PhD, Department of Laboratory Medicine and Pathobiology, Faculty of Medicine, University of Toronto, 1 King's College Circle, Toronto, ON, Canada M5S 1A8. E-mail: michael.ohh@utoronto.ca
} 
glucose transporter-1 (GLUT1) and erythropoietin (EPO). ${ }^{9}$ These discoveries have confirmed VHL as a critical regulator of the ubiquitous mammalian oxygen-sensing pathway.

HIF $\alpha$ is overexpressed in most tumors including CC-RCC. The re-introduction of VHL into VHL-null CC-RCC abrogates the tumorigenic potential of these cells in a mouse xenograft model. ${ }^{10,11}$ Kondo et al ${ }^{12}$ have shown that the expression of a non-degradable form of HIF $2 \alpha$ was able to restore the tumorigenic phenotype in CC-RCC cells ectopically expressing VHL. Conversely, shRNA-mediated knockdown of HIF $2 \alpha$ was sufficient to suppress the tumorigenic capacity of VHL-null CC-RCC cells. ${ }^{13}$ Notably, the emerging evidence suggests that VHL mutations affecting HIF regulation were invariably associated with a subtype of VHL disease with a greater propensity of CC-RCC. Although these reports support the notion that deregulated accumulation of HIF $\alpha$ upon the loss of VHL is crucial for the development of CC-RCC, the precise downstream target(s) of HIF that are responsible for renal epithelial oncogenesis have, until recently, remained unclear.

The regulation of adhesive interactions between cells is critical during cell growth and differentiation, and the loss of cell-cell adhesion is frequently associated with tumor progression and metastasis. ${ }^{14}$ Recently, VHL was shown to promote the transcription of E-cadherin via HIF-dependent activation of E-cadherin-specific transcriptional repressors. ${ }^{15-17}$ Thus, a loss of VHL in CC-RCC results in the hyperactivation of HIF that triggers the expression of E-cadherin repressors, which in turn attenuates the expression of E-cadherin. ${ }^{15-17}$ E-cadherin, a homophilic adhesion molecule associated with catenins that functions as a major component of cell junctions in polarized epithelial cells, is an established tumor suppressor. The graded loss of E-cadherin correlates with the aggressiveness of numerous carcinomas and the worsening of prognosis, while forced expression of E-cadherin suppresses tumor development and invasion in various in vitro and in vivo tumor model systems. ${ }^{14}$

The full-length E-cadherin $(120 \mathrm{kDa})$ can be proteolytically cleaved at a cleavage site near the transmembrane domain, which releases an extracellular $\mathrm{N}$-terminal $80-\mathrm{kDa}$ fragment and generates a $38-\mathrm{kDa}$-terminal fragment that can be further processed into a 33-kDa soluble cytosolic fragment. ${ }^{18}$ The proteolytic ectodomain release or 'shedding' of E-cadherin is emerging as an important regulatory mechanism and has been suggested to cause rapid changes in cell adhesion, signaling and apoptosis. ${ }^{19-22}$ Furthermore, increased levels of the soluble N-terminal fragment have been associated with several tumors, including prostate, ${ }^{23}$ gastric, ${ }^{24}$ hepatocellular $^{24}$ and bladder cancer and may be of prognostic value. ${ }^{21}$ Whether the C-terminal intracellular fragment of E-cadherin has oncogenic roles, for example, via promoting $\beta$-cateninmediated signaling, is unclear.

Here, we examined the prognostic value of VHL and E-cadherin in CC-RCC. While CC-RCC with negative E-cadherin staining exhibited no VHL immunoreactivity as expected, a significant portion of CC-RCC had aberrant nuclear E-cadherin staining despite positive VHL staining. DNA sequencing revealed in a majority of the nuclear E-cadherin-positive CC-RCC, subtle point mutations, deletions and insertions in VHL. The nuclear E-cadherin was not observed in chromophobe or papillary RCC, suggesting this to be specific to CC-RCC. Low Fuhrman grade and better prognosis associated with positive VHL and nuclear E-cadherin immunoreactivity, whereas high-grade tumors associated with a lack of nuclear E-cadherin staining and lower frequency of VHL staining. These findings provide the first evidence of an aberrant nuclear localization of E-cadherin in CC-RCC harboring VHL mutations, and suggest potential prognostic value of VHL and E-cadherin status in CC-RCC.

\section{MATERIALS AND METHODS Cell Culture}

Human 786-O CC-RCC cells were cultured in Dulbecco's modified Eagle's medium containing 10\% heat-inactivated fetal bovine serum (Sigma, Saint Louis, MI, USA) at $37^{\circ} \mathrm{C}$ in a humidified $5 \% \mathrm{CO}_{2}$ atmosphere. $786-\mathrm{O}$ subclones stably expressing hemagglutinin (HA)-tagged wild-type VHL30, HA-VHL19(WT), HA-VHL19(MUT) with V84M and P192Q mutations, or empty plasmid were generated as previously described. ${ }^{25,26}$

\section{Antibodies}

Anti-HA (12CA5; Roche Applied Science, Basel, Switzerland), anti-TCP1 (Abcam Inc., Cambridge, MA, USA), C-terminal-specific anti-E-cadherin (BD Biosciences, Mississauga, ON, Canada), N-terminal-specific anti-E-cadherin (Vector laboratories, Burlingame, CA, USA), anti-VHL (IG32) (BD Biosciences, Mississauga, ON, Canada), and anti- $\beta$-catenin (BD Biosciences, Mississauga, ON, Canada) monoclonal antibodies and anti-Glut1 polyclonal antibodies (Alpha Diagnostic International Inc., San Antonio, TX, USA) were obtained from the companies indicated.

\section{Plasmids}

The mammalian expression plasmid pRc-CMV-HA-VHL30 was previously described. ${ }^{25,26}$ pRc-CMV-HA-VHL19(WT) was generated from the pRc-CMV-HA-VHL30 plasmid using primer set 5'-CCCAAGCTTATGTATCCATATGATGTTCCA GATTATGCTGAGGCCGGGCGGCCG-3' ${ }^{\prime} / 5^{\prime}$-GCTCTAGATC AATCTCCCATCCGTTGATGTGC-3'. pRc-CMV-VHL19(MUT), V84M and P192Q, was serendipitously generated from pRcCMV-HA-VHL30 using the above primers in a PCR reaction. The authenticity of the plasmids was confirmed by direct DNA sequencing.

\section{Immunoprecipitation and Immunoblotting}

Cells were lysed in EBC buffer (50 mM Tris (pH 8), $120 \mathrm{mM}$ $\mathrm{NaCl}, 0.5 \% \mathrm{NP}-40$ ) supplemented with protease and phosphatase inhibitors (Roche, Laval, QC, Canada). Samples were supplemented with the indicated antibody and immobilized 
on protein A-Sepharose beads (Amersham Biosciences, Uppsala, Sweden). The samples were then washed five times with NETN buffer (20 mM Tris ( $\mathrm{pH} 8$ ), $120 \mathrm{mM} \mathrm{NaCl}, 1 \mathrm{mM}$ EDTA, $0.5 \%$ NP-40), eluted by boiling in sodium dodecyl sulfate (SDS)-containing sample buffer, separated by SDSpolyacrylamide gel electrophoresis (PAGE) and electrotransferred to a polyvinylidene difluoride membrane (Bio-Rad, Mississauga, ON, Canada). Specific protein bands on Western blots were visualized using the various antibodies indicated. All primary antibodies were diluted in phosphatebuffered saline (PBS) containing $0.1 \%$ Tween 20. Horseradish peroxidase-conjugated goat anti-mouse, goat anti-rabbit or rabbit anti-goat antibody (Pierce, Rockford, IL, USA) was used at 1:20 000 dilution as a secondary antibody, after which enhanced chemiluminescence (PerkinElmer Life Sciences, Woodbridge, ON, Canada) was performed for detection.

\section{Metabolic Labeling}

Metabolic labeling was performed as previously described. ${ }^{27}$ Briefly, radioisotope labeling was performed by methionine starvation for $45 \mathrm{~min}$, followed by growth in $5 \mathrm{ml}$ of methionine-free Dulbecco's modified Eagle's medium supplemented with $\left[{ }^{35} \mathrm{~S}\right]$ methionine $(100 \mu \mathrm{Ci} / \mathrm{ml}$ of medium; PerkinElmer Life Sciences, Woodbridge, ON, Canada) and $2 \%$ dialyzed fetal bovine serum at $37^{\circ} \mathrm{C}$ in a humidified $5 \%$ $\mathrm{CO}_{2}$ atmosphere for $3 \mathrm{~h}$ for the interaction studies and $1 \mathrm{~h}$ for half-life analysis.

\section{RNA Extraction}

RNA was extracted from cells using RNeasy kit according to the manufacturer's instructions (Qiagen, Mississauga, ON, Canada).

\section{Quantitative Real-Time PCR}

Quantitative real-time PCR was performed as previously described. ${ }^{28}$ Briefly, first-strand cDNA synthesis was performed as follows: $1 \mu \mathrm{l}$ of oligo $(\mathrm{dT})_{23}$ primer (Sigma, St Louis, MI, USA) was incubated with $5 \mu \mathrm{g}$ of RNA and $\mathrm{dH}_{2} \mathrm{O}$ (total reaction volume was $20 \mu \mathrm{l}$ ) for $10 \mathrm{~min}$ at $70^{\circ} \mathrm{C}$ in a thermal cycler (MJ research, Boston, MA, USA). The mixture was cooled to $4^{\circ} \mathrm{C}$, at which time $4 \mu \mathrm{l}$ of $5 \times$ first-strand reaction buffer, $2 \mu \mathrm{l}$ of $0.1 \mathrm{M} \mathrm{DTT}, 1 \mu \mathrm{l}$ of $10 \mathrm{mM}$ dNTPs and $1 \mu \mathrm{l}$ Superscript II reverse transcriptase (Invitrogen, Carlsbad, CA, USA) were added. cDNA synthesis was performed for $1.5 \mathrm{~h}$ at $42^{\circ} \mathrm{C}$, followed by $15 \mathrm{~min}$ at $70^{\circ} \mathrm{C}$ in the thermal cycler. Human genomic DNA (Roche, Mannheim, Germany) standards or cDNA equivalent to $20 \mathrm{ng}$ of total RNA were added to the qPCR reaction in a final volume containing $1 \times$ PCR buffer (without $\mathrm{MgCl}_{2}$ ), $3 \mathrm{mM} \mathrm{MgCl}, 0.25 \mathrm{U}$ Platinum Taq DNA polymerase, $0.2 \mathrm{mM}$ dNTPs, $0.3 \mu \mathrm{l}$ SYBR Green I, $0.2 \mu \mathrm{l}$ ROX reference dye and $0.5 \mu \mathrm{M}$ primers (Invitrogen, Carlsbad, CA, USA). Amplification conditions were as follows: $95^{\circ} \mathrm{C}(3 \mathrm{~min}) ; 40$ cycles of $95^{\circ} \mathrm{C}(10 \mathrm{~s}), 65^{\circ} \mathrm{C}(15 \mathrm{~s}), 72^{\circ} \mathrm{C}$ $(20 \mathrm{~s}), 60^{\circ} \mathrm{C}(15 \mathrm{~s})$ and $95^{\circ} \mathrm{C}(15 \mathrm{~s}) . \mathrm{qPCR}$ was performed using the ABI Prism 7900HT Sequence Detection System
(Applied Biosystems, Foster City, CA, USA). Gene-specific oligonucleotide primers designed using Primer Express (Applied Biosystems) were as follows: GLUT-1 primer set 1 (5'-CACCACCTCACTCCTGTTACTTACCT- $3^{\prime}$ and $5^{\prime}$-CAAG CATTTCAAAACCATGTTTCTA- ${ }^{\prime}$ ), GLUT-1 primer set 2 $\left(5^{\prime}\right.$-CTCCCAGCAGCCCTAAGGAT- $3^{\prime}$ and $5^{\prime}$-ATCTGTCAG GTTTGGAAGTCTCATC- $\left.3^{\prime}\right)$ and $\beta$-actin primer set $\left(5^{\prime}\right.$-GGA TCGGCGGCTCCAT- $3^{\prime}$ and $5^{\prime}$-CATACTCCTGCTTGCTGAT CCA-3 ${ }^{\prime}$ ). SYBR I fluoresces during each cycle of the qPCR by an amount proportional to the quantity of amplified cDNA (the amplicon) present at that time. The point at which the fluorescent signal is statistically significant above the background is defined as the cycle threshold $\left(C_{\mathrm{t}}\right)$. Expression levels of the various transcripts were determined by taking the average $C_{\mathrm{t}}$ value for each cDNA sample performed in triplicate, and measured against a standard plot of $C_{\mathrm{t}}$ values from amplification of serially diluted human genomic DNA standards. Since the $C_{\mathrm{t}}$ value is inversely proportional to the $\log$ of the initial copy number, the copy number of an experimental mRNA can be obtained from linear regression of the standard curve. A measure of the fold difference in copy number was determined for each mRNA. Values were normalized to the expression of $\beta$-actin mRNA and represent the average value of both GLUT-1 primers in three independent experiments performed in triplicate.

\section{In Vitro Ubiquitination Assay}

An in vitro ubiquitination assay was performed as previously described. ${ }^{29}$ The $\left[{ }^{35} \mathrm{~S}\right]$ methionine-labeled reticulocyte lysate HA-HIF- $1 \alpha$ ODD translation product $(4 \mu \mathrm{l})$ was incubated in 786-O RCC S100 extracts (100-150 $\mu \mathrm{g})$ supplemented with $8 \mu \mathrm{g} / \mu \mathrm{l}$ ubiquitin (Sigma), $100 \mathrm{ng} / \mu \mathrm{l}$ ubiquitin aldehyde (Boston Biochem Inc., Cambridge, MA, USA) and an ATPregenerating system (20 mM Tris (pH 7.4), $2 \mathrm{mM}$ ATP, $5 \mathrm{mM}$ $\mathrm{MgCl}_{2}, 40 \mathrm{mM}$ creatine phosphate and $0.5 \mu \mathrm{g} / \mu \mathrm{l}$ creatine kinase) in a reaction volume of $20-30 \mu$ l for $1.5 \mathrm{~h}$ at $30^{\circ} \mathrm{C}$.

\section{Protease Digestion Assay}

Protease sensitivity was assayed as previously described. ${ }^{30}$ Briefly, lysates were incubated with chymotrypsin $(10 \mu \mathrm{g} / \mathrm{ml})$ for $10 \mathrm{~min}$ on ice. Reactions were stopped by the addition of $1 \mathrm{mM}$ PMSF before immunoprecipitation (IP) and SDS-PAGE.

\section{SCID Mouse Xenograft Assay}

Multiple 786-O subclones expressing HA-VHL30, HAVHL19(MUT) or empty plasmid were grown to $\sim 90 \%$ confluence in a humidified $5 \% \mathrm{CO}_{2}$ atmosphere at $37^{\circ} \mathrm{C}$. Cells were harvested with a solution of $0.25 \%$ trypsin and $1 \mathrm{mM}$ EDTA. Cells $\left(1 \times 10^{7}\right)$ in $100 \mu \mathrm{l}$ of $1 \times$ PBS were injected intramuscularly into the left hind legs of male SCID mice (Charles River Laboratories Inc., Wilmington, MA, USA). Tumor growth was assessed and measured weekly by carefully passing the tumor-bearing leg through a series of 
holes of decreasing diameter (0.5-mm decrements) in a plastic rod.

\section{Immunohistochemistry}

SCID mouse xenograft tumors were fixed in 10\% neutral buffered formalin and later embedded in paraffin. Formalinfixed, paraffin-embedded sections from 19 nephrectomy specimens with CC-RCC were obtained from the Department of Pathology and Laboratory Medicine at The University Health Network (Toronto, ON, Canada). These tissue blocks were used and processed in accordance with a University Health Network Research Ethics Board-approved protocol concerning gene expression in RCC. Tissues were fixed in 10\% neutral buffered formalin for $24-36 \mathrm{~h}$. Representative sections of tumor with adjacent non-tumor renal parenchyma, 3-4 $\mathrm{mm}$ in thickness, were embedded in paraffin and 5- $\mu \mathrm{m}$ sections were cut and placed on coated slides for light microscopy.

Tumor morphology and classification were assessed using standard hematoxylin and eosin (H\&E) staining. The tumors were classified as conventional clear-cell type according to criteria described in the WHO classification of renal tumors. ${ }^{31}$ Immunohistochemical staining for VHL was performed manually using a standard avidin-biotin-peroxidase complex method. Briefly, unlabeled anti-HA, anti-Glut1, anti-E-cadherin (BD biosciences, Mississauga, ON, Canada), anti-E-cadherin (Vector laboratories, Burlingame, CA, USA) and anti- $\beta$-catenin were used at a 1:2000 dilution, and an overnight incubation following microwave pretreatment for antigen retrieval. The sections were then incubated with a biotinylated secondary antibody (horse anti-mouse IgG, 1:200 dilution) and the avidin-peroxidase complex. The colored reaction was visualized using nova red as the chromogen. The tissue was then mildly counterstained with hematoxylin.

\section{Tissue Microarray}

Tissue microarray (TMA) consisting of quadruplicate representative $1.0-\mathrm{mm}$ cores from 56 CC-RCC with various Fuhrman grades (grades 1-4) and stages (pT1-pT4) were used to analyze the correlation between E-cadherin and VHL protein expression. Sections $(5 \mu \mathrm{m})$ from the CC-RCC TMA were stained with anti-E-cadherin and anti-VHL antibodies as described above. The slides were then scanned using a ScanScope (Aperio Technologies, Vista, CA, USA) and scored by two observers. In order to be considered for evaluation, at least half of the TMA core was required to be present on the slide, with at least $50 \%$ of the tissue present being tumor cells. Cores were being scored as either positive or negative. Only tumors with the majority of cores in agreement ( 3 out of 4 or 2 out of 3 ) were used for further analysis.

\section{VHL Sequencing}

VHL was amplified from patient samples using primers 5'-CAGTAACGAGTTGGCCTAGC-3'/5'-GGATGTGTCCTG
CCTCAAG-3' for exon 1, 5'-GGACGGTCTTGATCTCCTGA$3^{\prime} / 5^{\prime}$-GATTGGATACCGTGCCTGAC-3' for exon 2 and $5^{\prime}$-CT GCCACATACATGCACTCA- $3^{\prime} / 5^{\prime}$-AAGGAAGGAACCAGTCC TGT- $3^{\prime}$ for exon 3. PCR reactions were performed using the Qiagen Multiplex Master Mix (Qiagen, Mississauga, ON, Canada) according to manufacturer's instructions. Exons were sequenced with the Cy5/Cy5.5 Dye Primer Cycle Sequencing kit (Amersham, Quebec/Visible Genetics, Toronto) according to the manufacturer's instructions. Each primer mixture contained two primers, labeled with either Cy5 or Cy5.5. Primers used for sequencing were $5^{\prime}$-/5Cy5/-CTAGCCTCGCCTCCGT TA-3'/5'-/5Cy5/-GCCTCAGTTCCCCGTCTG-3' for exon 1, $5^{\prime}$-/5Cy5-GATACCGTGCCTGACATCAG-3' for exon 2 and 5'-/5Cy5/-CAGGTAGTTGTTGGCAAAGC-3' for exon 3. Two primers were used for exon 1 as it is a large exon. The sequences generated were compared with wild-type VHL (GenBank accession number AC_000046) for sequence alterations, using the OpenGene Automated DNA System and Gene Librarian software, version 3.1 (Visible Genetics, Suwanee, GA, USA).

\section{Statistics}

Fisher's exact test was used to determine whether positive VHL immunoreactivity and nuclear E-cadherin subcellular localization were interdependent. Two-tailed $P$-values less than 0.05 were considered significant.

\section{RESULTS \\ Nuclear Localization of E-cadherin is Associated with CC-RCC-Harboring VHL Mutation}

VHL acts as the substrate-recognition moiety of an E3 ubiquitin ligase that ubiquitylates $\mathrm{HIF} \alpha$ for $26 \mathrm{~S}$ proteasomemediated destruction. Recently, we and others have shown that upon the loss of VHL, HIF negatively regulates the transcription of E-cadherin by upregulating the transcriptional repressors of E-cadherin. ${ }^{15-17}$ Consequently, CC-RCC cells devoid of VHL fail to express E-cadherin. ${ }^{15-17}$ Although CC-RCC on TMA with negative VHL immunoreactivity correlated with negligible E-cadherin staining as predicted, a significant portion $(49 \%$ or $22 / 45)$ of the samples that met the quality standard criteria (see Materials and Methods) showed previously undocumented nuclear E-cadherin localization (Figure 1a, left panel). The majority ( $77 \%$ or $17 / 22)$ of these 22 nuclear E-cadherin-positive samples stained positive, while $14 \%(3 / 22)$ negative and $9 \%(2 / 22)$ uncertain for VHL, indicating a statistically significant correlation between nuclear E-cadherin staining and positive VHL immunoreactivity $(P=0.009)$ (Figure 1a, right panel). Of the 23 CC-RCC samples that were negative for nuclear E-cadherin, $39 \%(9 / 23)$ were positive, $48 \%(11 / 23)$ were negative and $13 \%(3 / 23)$ were inconclusive for VHL immunoreactivity.

The vast majority (approximately $80 \%$ ) of CC-RCC have VHL mutations. Thus, a positive VHL immunostaining would not necessarily indicate a wild-type VHL status. We next determined via direct DNA sequencing the VHL status 
a

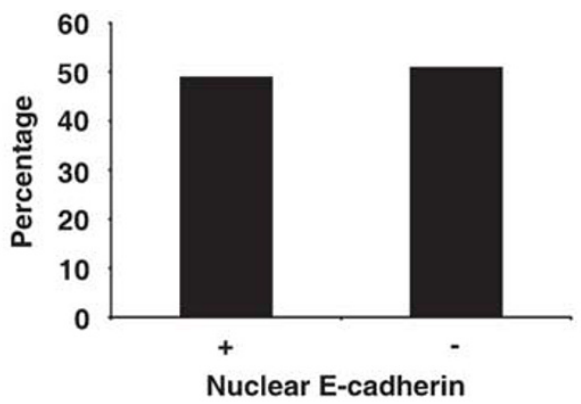

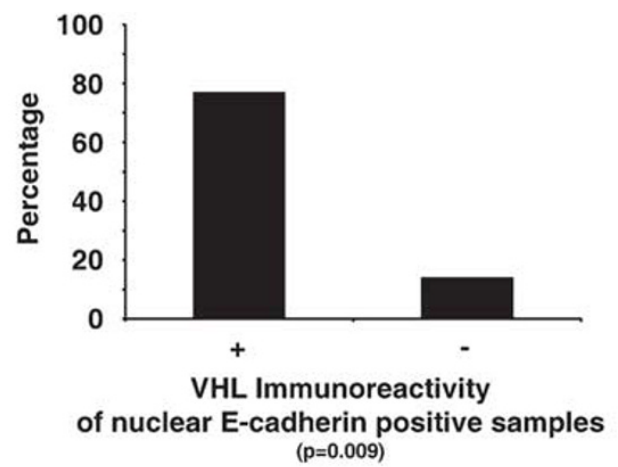

$(p=0.009)$

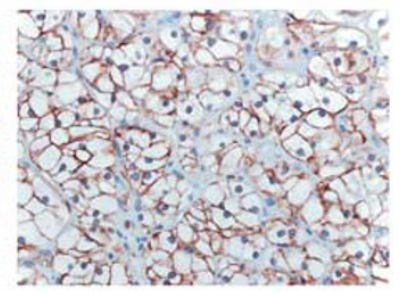

VHL(F136S)

C

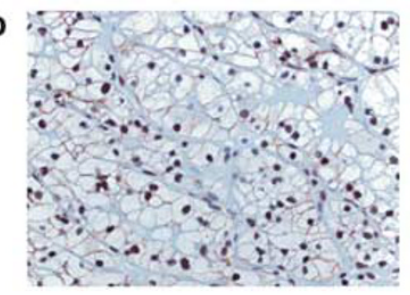

C-term E-cad

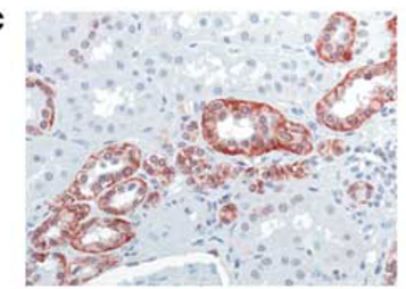

Normal Kidney

C-term E-cad
N-term E-cad
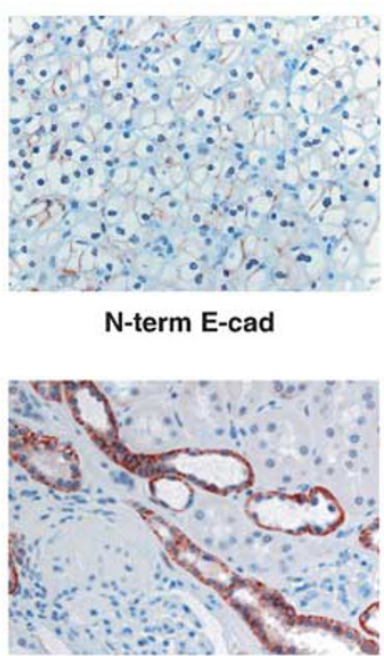

Normal Kidney

$\mathrm{N}$-term E-cad

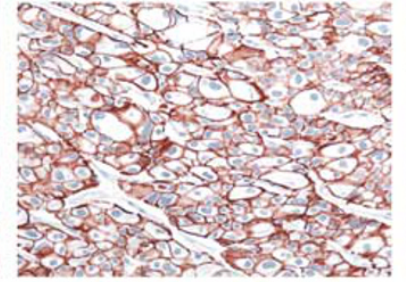

Chromophobe RCC C-term E-cad

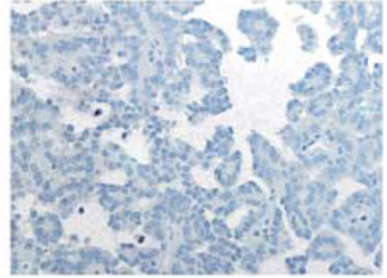

Papillary RCC

C-term E-cad

Figure 1 Nuclear localization of E-cadherin correlates with positive VHL immunostaining in CC-RCC. (a) CC-RCC TMA were immunostained with antiE-cadherin antibody and scored for nuclear E-cadherin expression (left panel). Nuclear E-cadherin-positive CC-RCC samples were then scored for VHL immunoreactivity (right panel). (b) Immunohistochemical staining of a representative tumor tissue sample (patient no. 9) with VHL mutation using the indicated antibodies. (c) Immunohistochemical staining of a representative normal kidney tissue sample (patient no. 9), as well as representative samples on TMA slide constructed using quadruplicate samples from chromophobe and papillary RCC subtypes using the indicated antibodies. Notably, chromophobe RCC is known to express E-cadherin, while papillary RCC has been shown to be a non-expresser of E-cadherin. ${ }^{32}$

of CC-RCC samples with positive nuclear E-cadherin staining. Seventeen of the 22 nuclear E-cadherin-positive CC-RCC samples were available for $V H L$ sequencing. Of these 17 samples, 13 stained positive for VHL, two were negative and two had indeterminate VHL staining. Consistent with the prevalence of VHL inactivation in CC-RCC, we found VHL mutations in $62 \%(8 / 13)$ of CC-RCC samples that stained positively for VHL (Table 1). Therefore, a high percentage of CC-RCC with nuclear E-cadherin staining contained detectable VHL mutations despite positive VHL immunoreactivity. Moreover, we found that the two samples that had negative staining for VHL (sample number 18 and 19) harbored VHL mutations, indicating that these mutations may have affected the ability of VHL to be recognized by antiVHL antibody, and one of the two samples that had uncertain VHL staining (sample number 17) had VHL mutation. Although unable to establish statistical significance due to currently available small sample size, nuclear E-cadherin staining showed a positive trend with the presence of a VHL mutation. Notably, nuclear E-cadherin is likely a cleavage product of the membranous full-length E-cadherin (discussed below). In keeping with this notion, a significant portion of CC-RCC samples with nuclear E-cadherin staining also exhibited membranous E-cadherin staining (Table 1).

E-cadherin is a type I transmembrane glycoprotein that has been shown to be proteolytically cleaved in its transmembrane domain, resulting in an $80-\mathrm{kDa} \mathrm{N}$-terminal soluble fragment and an intracellular C-terminal fragment. ${ }^{21}$ We asked whether the observed nuclear E-cadherin in CCRCC is the full-length protein or a truncated form. TMA were immunostained with an anti-E-cadherin antibody generated against the $\mathrm{N}$-terminus or $\mathrm{C}$-terminus of E-cadherin. While both antibodies immunostained membranous E-cadherin, the nuclear E-cadherin was exclusively recognized by the C-terminus-specific anti-E-cadherin antibody, as illustrated 
Table 1 VHL mutational analysis of CC-RCC samples with positive nuclear E-cadherin staining

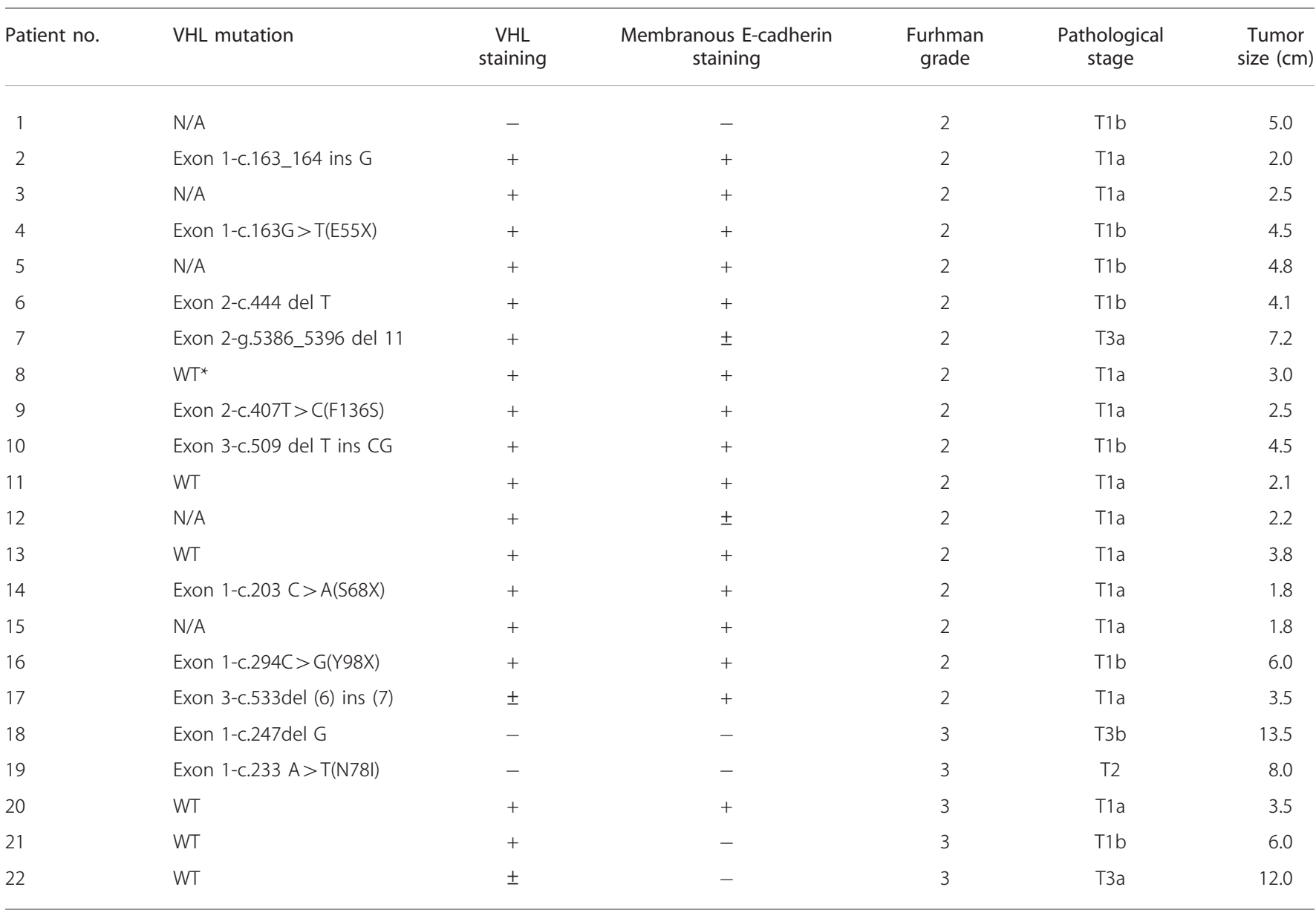

CC-RCC, clear-cell renal cell carcinoma; N/A, not available; VHL, von Hippel-Lindau.

*WT indicates wild-type; + indicates 3 or 4 cores out of four stained positive; \pm indicates 2 cores out of four stained positive; -indicates 0 or 1 core out of four stained positive.

in the representative Figure $1 \mathrm{~b}$ (and data not shown) representing a CC-RCC sample from patient no. 9 shown to have F136S substitution mutation in VHL (Table 1). Notably, the normal kidney tissue from patient no. 9 showed an archetypal membranous E-cadherin staining of the proximal tubule cells, regardless of the antibody used and, importantly, no nuclear localization of E-cadherin was observed (Figure 1c). We next asked if nuclear localization of E-cadherin is a phenomenon unique to CC-RCCs. We stained a TMA slide constructed using quadruplicate samples from chromophobe and papillary RCC subtypes with the C-terminal E-cadherin antibody that showed nuclear staining in the CC-RCC cases. Chromophobe RCC has been reported to express E-cadherin, while papillary RCC has been shown to be a non-expresser of E-cadherin. ${ }^{32}$ As expected, chromophobe RCC cells exhibited membranous E-cadherin immunoreactivity, but no nuclear protein was found. Papillary RCC was negative for E-cadherin immunoreactivity (Figure 1c). These results suggest that the aberrant nuclear localization of C-terminal E-cadherin is specific to RCC of clear-cell type.
The Synthetic VHL19 Mutant Exhibits the Biochemical Signature of a Disease-Prone Mutation

The molecular validation of critical pathways implicated in VHL-associated CC-RCC development and progression has been widely studied in immune-compromised Nude or SCID mice. We asked whether the aberrant nuclear localization of E-cadherin is recapitulated in human CC-RCC xenograft transplanted in SCID mice. VHL gene produces two wild-type forms, a full-length VHL30 and an internally translated VHL19. ${ }^{33-35}$ Although ectopic expression of either isoform has been shown to suppress CC-RCC tumor formation in Nude mice, ${ }^{10,11,33,35}$ demonstrating that both isoforms possess tumor suppressor functions, VHL19 is more abundantly produced than VHL30. Therefore, the general perception is that VHL19 is the dominant isoform, at least, in cell lines. However, mutations in the context of VHL19 have not been examined in a mouse xenograft assay.

No disease-causing mutation in the exclusive context of VHL19 has been reported. Thus, we generated 786-O 
CC-RCC subclones stably expressing HA-VHL19(MUT) with synthetic V84M and P192Q substitutions, which would simultaneously represent $\alpha$ - and $\beta$-domain-related VHL mutations based on the reported crystal structure of VHL19/elongin B/elongin C complex. ${ }^{36}$ Cells expressing HA-VHL19(MUT), HA-VHL19(WT) or empty plasmid were metabolically labeled with $\left[{ }^{35} \mathrm{~S}\right]$ methionine, lysed, immunoprecipitated with an anti-HA antibody and the bound proteins were resolved by SDS-PAGE (Figure 2a). In comparison with HA-VHL19(WT) or HA-VHL30(WT), which generated similar associated-protein profile as expected, ${ }^{37-39}$ HA-VHL19(MUT) was deficient in co-precipitating elongins $\mathrm{B} / \mathrm{C}$ and Cullin 2 (Cul2), the components of the ECV complex (Figure 2a; Supplementary Figure 1). Consistent with reduced elongin/Cul2 binding, 786VHL19(MUT) showed reduced HIF $\alpha$ (ODD) ubiquitylation in an in vitro ubiquitination assay and CC-RCC cells expression VHL19(MUT) had correspondingly increased level of HIF-target GLUT1 expression under normal oxygen tension (Supplementary Figure 2). Although there are conflicting reports with regards to VHL19-fibronectin interaction, possibly due to the heterogeneity of cultured CC-RCC cell lines, ${ }^{34,37}$ we found that HA-VHL19(WT) but not HA-VHL19(MUT) co-precipitated fibronectin (Figure 2a). Moreover, VHL30(WT) has previously been shown to associate with the eukaryotic chaperonin TRiC/CCT complex (see also Supplementary Figure 1). ${ }^{40-42}$ Proper TRiC-mediated folding of VHL30 is required for binding elongins $\mathrm{B} / \mathrm{C}$ and VHL disease-causing mutants have been shown to have elevated binding of the TRiC complex. ${ }^{40-42}$ Indicative of a mutant phenotype, HA-VHL19(MUT) showed markedly increased binding of the TRiC complex, including one of the components TCP1, than HA-VHL19(WT) or HA-VHL30(WT) (Figure 2a and b; Supplementary Figure 1). A consequence of increased TRiC interaction is the attenuated resistance to protease digestion, suggesting that a mutant VHL has a loose unfolded structure that is sensitive to proteases such as chymotrypsin and thermolysin. ${ }^{30}$ In accordance, HA-VHL30(WT) was resistant to protease digestion as expected, whereas HA-VHL19(MUT) was sensitive to chymotrypsin (Figure 2c). HA-VHL30(C162F) served as a positive control for protease sensitivity (Figure $2 c$ ), as this disease-causing mutant is incapable of binding elongin $\mathrm{C}$ and remains bound to the TRiC complex. $^{36,42}$ We next asked whether the sensitivity to protease digestion correlated with shortened half-life. 786-MOCK, 786-VHL30 and 786-VHL19(MUT) cells were pulsed with $\left[{ }^{35} \mathrm{~S}\right]$ methionine for $1 \mathrm{~h}$ (or treated with cyclohexamide; data not shown) and chased for the indicated number of hours (Figure 2d). As expected, HA-VHL30 had a long protein half-life, whereas HA-VHL19(MUT) had a dramatically shortened half-life (Figure 2d). These results taken together demonstrate that HA-VHL19(MUT) has all the biochemical signatures of a defective, disease-prone VHL mutation.

\section{CC-RCC Tumor Xenograft in SCID Mice Exhibits Nuclear E-Cadherin Expression}

As predicted from the biochemical analysis, stable expression of HA-VHL19(MUT) failed to suppress the tumor growth of 786-VHL19(MUT) cells in SCID mice (Figure 3a). VHLnull 786-MOCK subclones grew tumors, whereas 786VHL30(WT) cells did not form tumors, as expected (Figure 3a). The tumors were resected from the hind legs of mice, fixed in $10 \%$ neutral buffered formalin and embedded in paraffin. Sections $(5 \mu \mathrm{m})$ were placed on coated slides for standard H\&E staining (Figure 3b, first panel). Both the 786MOCK and 786-VHL19(MUT) tumors were characterized by nests and clusters of cells surrounded by a delicate microvascular network. This feature is characteristic of CC-RCC. ${ }^{31}$ The tumor cells in both xenografts had large, moderately pleomorphic nuclei with prominent nucleoli and variable amounts of pale eosinophilic cytoplasm. The nuclear features are consistent with those seen in high-grade (Fuhrman grade 3-4) RCC. They also contained foci in which the tumor cell nuclei adopted a spindleform morphology, indicative of sarcomatoid differentiation. 786-VHL19(MUT) tumors also stained positive for $\mathrm{HA}$ as predicted, indicating the presence of HA-VHL19(MUT) in tumor cells (Figure 3b, second panel), and both tumor types stained for GLUT1 expression (Figure 3b, third panel), which indicates a failure in the negative regulation of HIF in the VHL-null or VHL19 mutant cells.

In an effort to determine the affect of mutations in VHL19 on E-cadherin subcellular localization, 786-MOCK and 786VHL19(MUT) xenografts were stained with an anti-E-cadherin antibody specific to the C-terminus or N-terminus of E-cadherin (Figure 3b, fourth panel or fifth panel,

Figure 2 VHL19 mutant exhibits biochemical signature of a disease-prone mutation. (a) The indicated 786-O subclones were radiolabeled with $\left[{ }^{35} \mathrm{~S}\right]$ methionine, lysed and immunoprecipitated with anti-HA antibody. The bound proteins were resolved on SDS-PAGE and visualized by autoradiography. (b) The indicated 786-O subclones were lysed and immunoprecipitated with anti-HA antibody (lanes 1-3) or anti-TCP1 antibody (lane 4), and immunoblotted with an anti-TCP1 antibody (upper panel) or anti-HA antibody (lower panel). (c) The indicated 786-O subclones were lysed and treated with $10 \mu \mathrm{g} / \mathrm{ml}$ chymotrypsin for $10 \mathrm{~min}$ on ice. Reactions were stopped with $1 \mathrm{mM}$ PMSF and the lysates were immunoprecipitated with an anti-HA antibody. Bound proteins were resolved by SDS-PAGE and immunoblotted with an anti-HA antibody. (d) The indicated 786-O subclones were pulsed with $\left[{ }^{35} \mathrm{~S}\right]$ methionine for $1 \mathrm{~h}$, chased for the indicated times and then cell lysates were immunoprecipitated with an anti-HA antibody. Bound proteins were resolved by SDS-PAGE and visualized by autoradiography. Experiments were performed multiple times using several subclones with similar outcome.

* Represents nonspecific proteins. IP, immunoprecipitation; IB, immunoblot. 
respectively). Interestingly, both tumor types did not exhibit membranous E-cadherin but showed nuclear E-cadherin, which was exclusively stained by C-terminus-specific antiE-cadherin antibody. This is consistent with the C-terminally cleaved nuclear E-cadherin observed in CC-RCC samples on
TMA. However, the nuclear E-cadherin expression in 786-MOCK xenograft is inconsistent with CC-RCC cells with negative VHL status, which correlated with negligible E-cadherin expression with no detectable nuclear E-cadherin staining. Human colon tissue was used as a positive control,

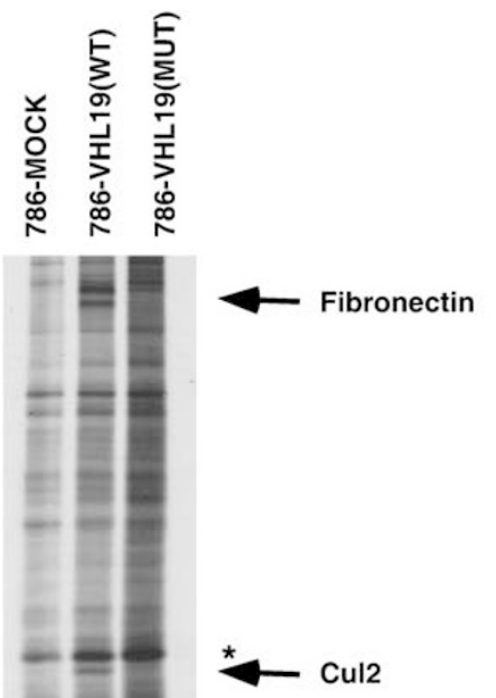

ᄀ TRiC/CCT

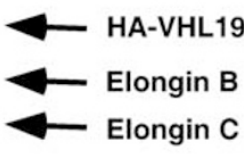

Anti-HA: IP b

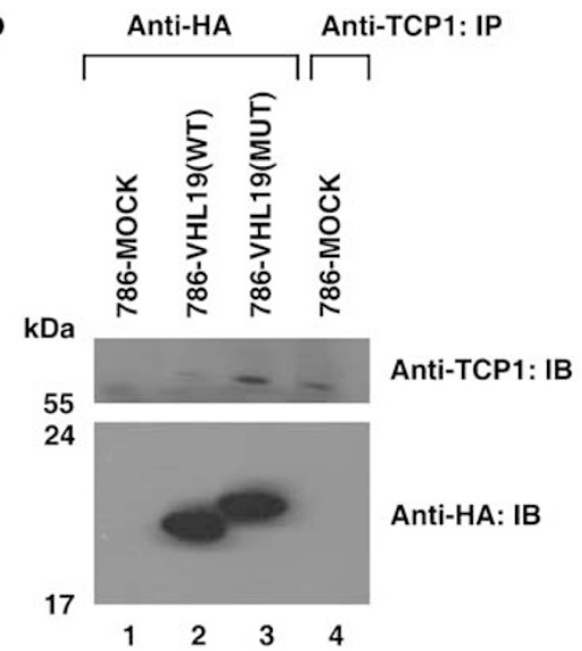

C

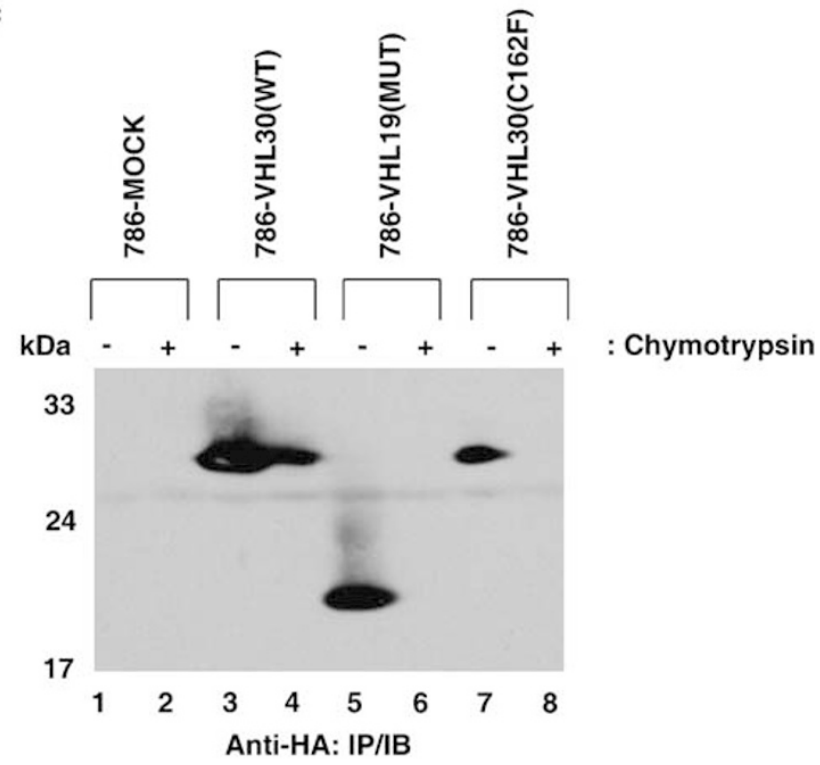

d

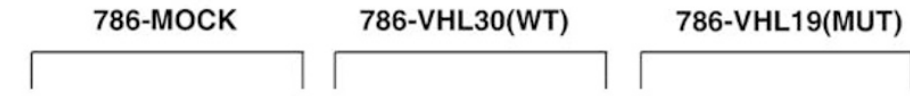

$\begin{array}{lllllllllllllll}0 & 1 & 3 & 5 & \mathrm{O} / \mathrm{N} & 0 & 1 & 3 & 5 & \mathrm{O} / \mathrm{N} & 0 & 1 & 3 & 5 & \mathrm{O} / \mathrm{N}\end{array}$

: h post-radiolabeling

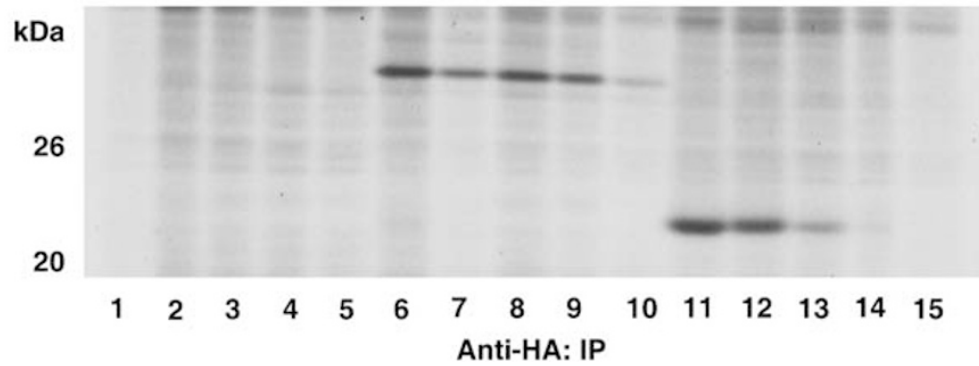




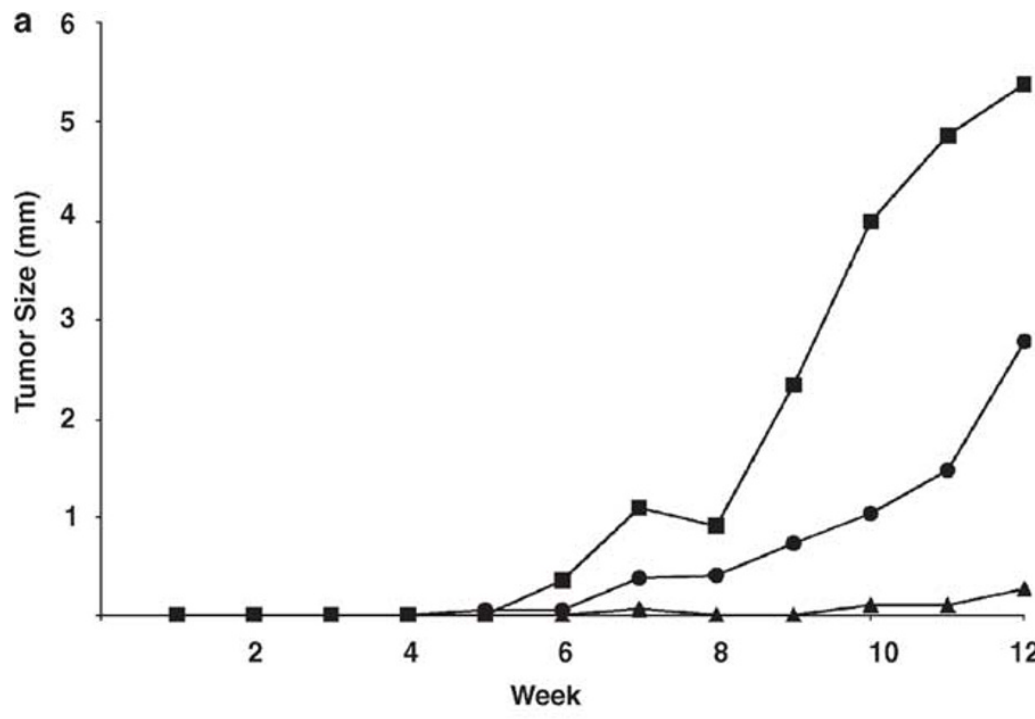

786-VHL19(MUT) (13/14)

b

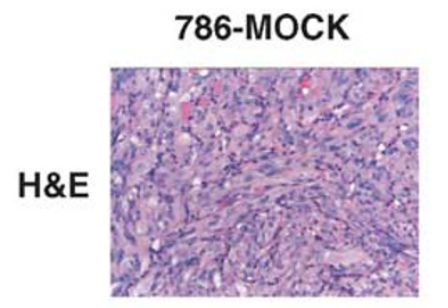

786-VHL19(MUT)

HA
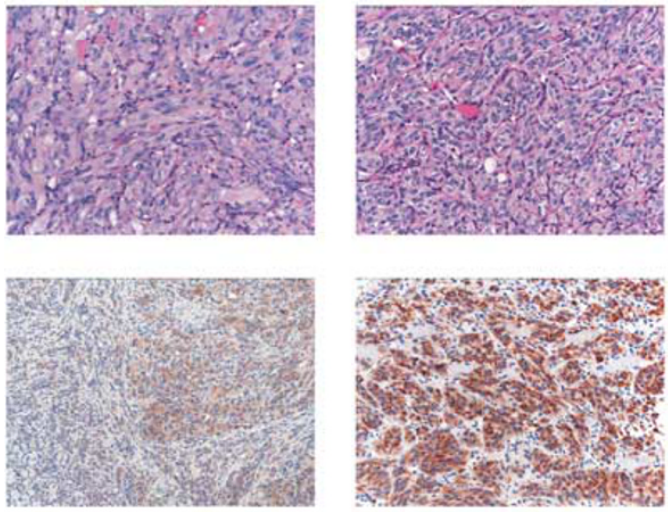

786-Mock (8/9)

786-VHL30(WT) (1/9)

Glut1
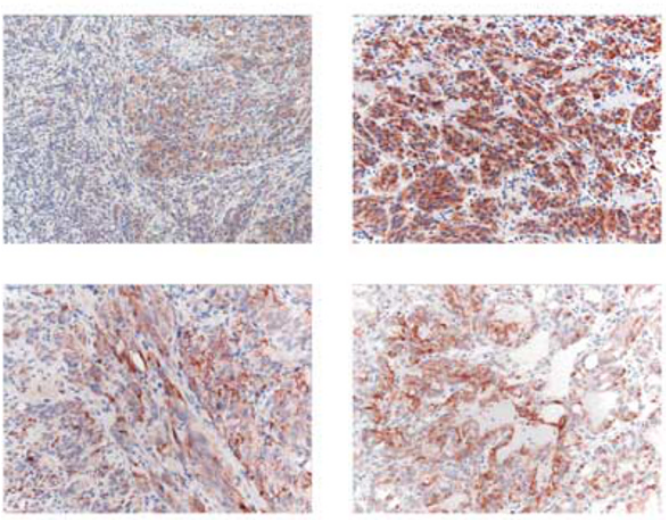

\section{E-cadherin (c-term)}
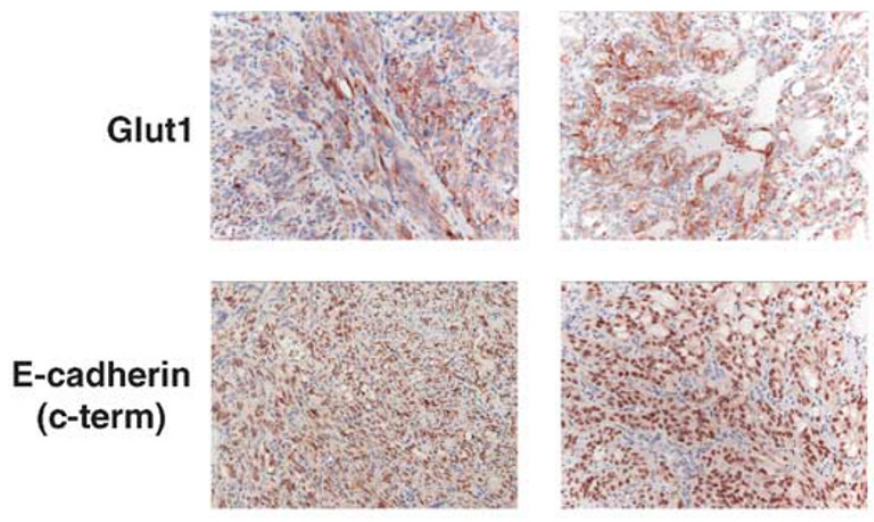

\section{E-cadherin (N-term)}
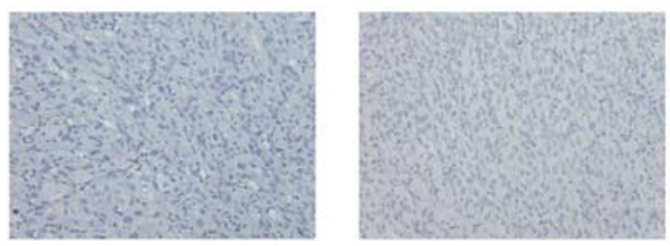

Figure 3 786-MOCK and 786-VHL19(MUT) xenografts in SCID mice display nuclear E-cadherin. (a) SCID mice were injected intramuscularly in the left hind leg with the indicated 786-O subclones and tumor size measured over time. The total number of mice that grew tumor is indicated in parentheses. This is a representative experiment of three independent experiments using multiple isogenic clones. (b) A representative photomicrographs of 786-MOCK and 786-VHL19(MUT) xenografts fixed and embedded in paraffin for the indicated immunohistochemical analysis. H\&E, hematoxylin and eosin staining. 


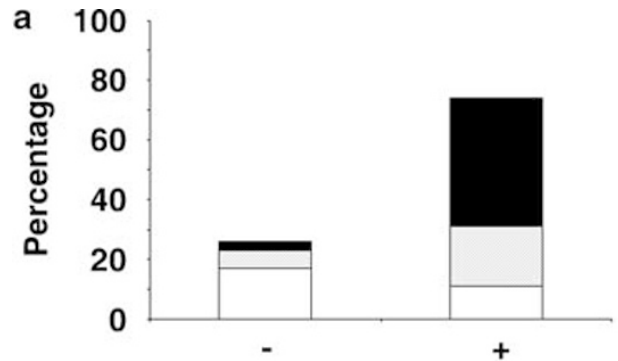

VHL Immunoreactivity in Low-Grade Tumours

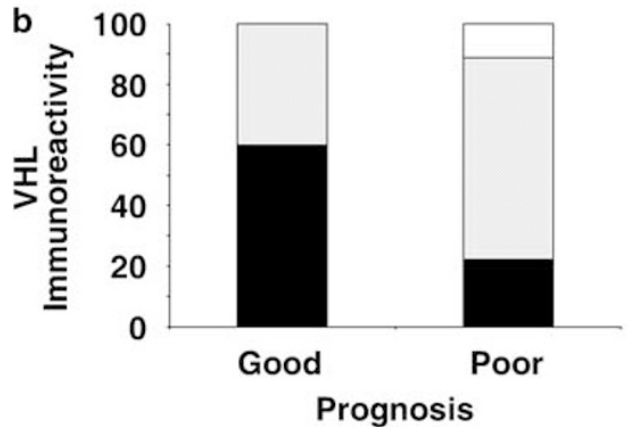

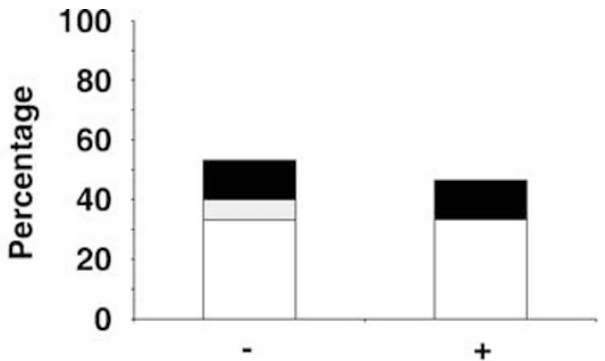

VHL Immunoreactivity in High-Grade Tumours

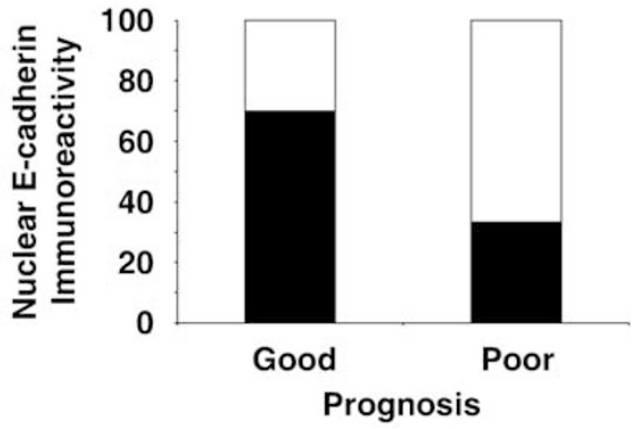

Figure 4 VHL immunoreactivity associates with low-grade tumors and better prognosis. (a) The CC-RCC samples on TMA were categorized as either low grade (Fuhrman grades 1 and 2) or high grade (Fuhrman grades 3 and 4) and scored for VHL and nuclear E-cadherin immunoreactivity. Solid bars represent the percentage of samples with positive nuclear E-cadherin staining. Open bars represent the percentage of samples with negative nuclear E-cadherin staining. Hatch bars represent the percentage of samples with indeterminate nuclear E-cadherin staining. (b) Representative sections from 19 CC-RCC were analyzed according to tumor behavior, based on clinical follow-up information. Good-prognosis cases were selected based on the absence of recurrent disease over 5 years of follow-up. Poor-prognosis cases were selected based on the presence of metastases at the time of presentation or development of metastases within 1 year of nephrectomy. VHL staining (left panel) for these sections was scored semi-quantitatively as being negative (open bars), weak (hatch) or strong (solid bars). Nuclear E-cadherin staining (right panel) was scored as either positive (solid bars) or negative (open bars).

which stained membranous E-cadherin using both E-cadherin antibodies (data not shown).

\section{VHL Immunoreactivity, which Correlates with Nuclear E-Cadherin, is Associated with Low-Grade Tumors and Better Prognosis}

Fifty CC-RCC samples on TMA met the quality standard criteria (see Materials and Methods) with regards to VHL immunoreactivity. Tumors were categorized as either low grade (Fuhrman grades 1 and 2) or high grade (Fuhrman grades 3 and 4). A total of 74\% (26/35) of low-grade tumors had positive VHL immunoreactivity, in contrast to $47 \%$ (7/15) of the high-grade tumors (Figure 4a). In addition, a high percentage $(46 \%$ or $16 / 35)$ of the low-grade tumor samples stained positive for nuclear E-cadherin, whereas only $29 \%(10 / 35)$ were nuclear E-cadherin negative. The remaining 26\% (9/35) had uncertain or inconclusive nuclear E-cadherin staining (ie, 2 out of 4 cores stained positive). Furthermore, of the 26 VHL-positive low-grade tumors, 15 exhibited nuclear E-cadherin expression while only four were negative, and the remaining seven were inconclusive of nuclear E-cadherin staining (Figure 4a). In contrast, a majority $(67 \%$ or $10 / 15)$ of the high-grade tumors showed negative nuclear E-cadherin staining. Five of those 10 were VHL positive and the other five VHL negative (Figure $4 \mathrm{a}$ ).

In an effort to assess the relationship between VHL immunoreactivity and tumor behavior, we selected representative sections from 19 CC-RCC according to tumor behavior based on clinical follow-up information. Specifically, good prognosis cases were selected based on the absence of recurrent disease over 5 years of follow-up. Poor prognosis cases were selected based on the presence of metastases at the time of presentation or development of metastases within 1 year of nephrectomy. VHL staining for these sections was scored semi-quantitatively as being negative, weak (focal or moderately diffused) or strong. A total of $60 \%(6 / 10)$ of the tumors in the good prognosis group showed strong VHL immunoreactivity, as compared with $22 \%(2 / 9)$ of the tumors from the poor-prognosis group (Figure 4b). Moreover, none of the good-prognosis tumors showed negative VHL staining and $4 / 10$ had weak staining, whereas $67 \%(6 / 9)$ of the poor-prognosis tumors showed only weak expression, including one case that was negative for VHL expression (Figure $4 \mathrm{~b}$ ). Furthermore, 70\% (7/10) of good-prognosis tumors had nuclear E-cadherin, whereas only 33\% (3/9) of poor-prognosis tumors had E-cadherin localized to the nucleus (Figure $4 \mathrm{~b}$ ). These results suggest that VHL 
immunoreactivity and E-cadherin expression/subcellular localization are associated with clinical outcome, such that there is a trend where higher Fuhrman grade or more aggressive CC-RCC have reduced VHL and E-cadherin immunoreactivity. CC-RCC with preserved VHL immunoreactivity, but a VHL mutation, associate with E-cadherin localized to both the membrane and the nucleus, and with low-grade tumors with more favorable clinical behavior.

\section{DISCUSSION}

Enhanced ability to predict tumor behavior in patients with CC-RCC will result in better selection of established and novel therapies. In this study we examined 45 patient CCRCC samples for VHL and E-cadherin immunoreactivity, and 50 samples for Fuhrman grade and VHL immunoreactivity. CC-RCC samples that stain positive for VHL are associated with better prognosis and low Fuhrman grade, whereas CC-RCC samples devoid of VHL expression have worse clinical outcome and high Fuhrman grade. In addition, nuclear localization of E-cadherin is correlated with positive, albeit mutant, VHL immunoreactivity. Therefore, CC-RCC can be subdivided into two groups where the first group is associated with the loss of VHL and E-cadherin expression and likely have poor prognosis. The second subset has positive VHL immunoreactivity correlating with nuclear E-cadherin localization and likely have better prognosis. Despite the positive staining of VHL in this subset, direct DNA sequencing revealed VHL mutations in the vast majority of CC-RCC samples. This is also consistent with the report by Yao et al ${ }^{43}$ demonstrating that the presence of VHL alteration is associated with better outcome for patients with stage I-III CC-RCC.

Although this is the first report of nuclear E-cadherin in CC-RCC, aberrant staining of E-cadherin in the nucleus has been reported in other types of cancer, including Merkel cell carcinoma, signet ring cell carcinoma, and solid pseudopapillary tumors (SPT) of the pancreas. ${ }^{44-46}$ The cytoplasmic domain of $\mathrm{E}$-cadherin is in a complex with $\beta$-catenin, implicating a potential 'outside-in' signaling, where a loss of E-cadherin or cleavage of E-cadherin releasing the intracellular fragment of E-cadherin would promote nuclear accumulation of $\beta$-catenin to associate with the leukocyte enhancer factor (LEF)/T-cell factor (TCF) to regulate the transcription of cell cycle (eg, cyclin D1) or invasion-related genes (eg, metalloproteinase matrilysin). ${ }^{14}$ Interestingly, an increased level of cyclin D1 has been observed in RCC cells devoid of VHL at high cell density. ${ }^{47}$ Whether the nuclear localization of E-cadherin in CC-RCC is associated with increased nuclear $\beta$-catenin is currently unknown. Furthermore, gastric and colorectal signet ring cell carcinomas and SPT with nuclear E-cadherin showed increased $\beta$-catenin levels in the nucleus. ${ }^{44,46}$ However, an expression of nuclear E-cadherin is not an invariable predictor of nuclear $\beta$-catenin, as the nuclear localization of E-cadherin in Merkel cell carcinoma was not associated with increased nuclear $\beta$-catenin expression. ${ }^{45}$

Although the general morphology and the microvasculature of the CC-RCC xenografts in SCID mice were reminiscent of patient CC-RCC, the nuclear features were more like those seen in high grade/sarcomatoid RCC. The apparently aggressive morphologic features seen in these grafts may be a result of repeated subculturing and 'phenotypic drift' over time. This phenomenon may well account for the discrepancies observed between the xenografts and patient samples used in our study. Notably, while CC-RCC xenografts in SCID mice recapitulated nuclear E-cadherin expression profile, nuclear E-cadherin staining was also observed in VHL-null CC-RCC xenografts, which is different from human tumor samples where CC-RCC with negative VHL staining lacked E-cadherin expression.

Proper regulation of cell-cell adhesion is crucial for growth, differentiation and development. The deregulation of cell adhesion leading to a disruption in cell anchorage can lead to pathological processes including tumor invasion and metastasis caused by alterations in cell movement and signaling. ${ }^{21}$ E-cadherins are $\mathrm{Ca}^{2+}$-dependent homophilic adhesion molecules that are associated with catenins and are major members of adherens junctions in polarized epithelial cells. E-cadherin has been shown to be regulated by several different mechanisms, including post-translational modification and regulation of gene expression. ${ }^{21,48}$ Proteolytic ectodomain release of transmembrane receptors, such as E-cadherin, is proving to be an important mechanism in regulating signaling functions of the cell surface proteins, resulting in the aberrant cell adhesion and altered intracellular signaling. ${ }^{21}$ Release of the E-cadherin- $\beta$-catenin complex from the membrane has previously been shown to result in elimination of cell-cell junctions. ${ }^{49}$ Furthermore, several type I transmembrane molecules have been shown to translocate to the nucleus following cleavage, including Notch, ErbB-4 and $\gamma$-protocadherin. ${ }^{50-52}$ For example, the translocation of Notch to the nucleus promotes Notchmediated activation or repression of target genes through its interaction to transcription factors. ${ }^{53}$ Although the function of nuclear E-cadherin is unknown, it is possible that like Notch, the E-cadherin cleavage product might influence the transcription of target genes when it localizes to the nucleus.

The soluble cleaved form of E-cadherin has been observed in serum under non-pathological conditions, but increased levels of the soluble form have been detected in patient peripheral blood samples ${ }^{24,54}$ and urine samples ${ }^{55}$ in a variety of diseases. ${ }^{21}$ It has been suggested that the $80-\mathrm{kDa}$ soluble fragment may serve as a useful biomarker of disease progression for a number of cancers, including prostate, ${ }^{23}$ gastric, ${ }^{24}$ hepatocellular ${ }^{24}$ and bladder cancer. ${ }^{55}$ There has also been a correlation between tumor stage, histological grade and elevated levels of soluble E-cadherin, likely because the detection of soluble E-cadherin has been associated with increased invasiveness. ${ }^{23,56,57}$ Although reasonable, it is 
currently unknown whether the detection of the C-terminal fragment of E-cadherin in the nucleus of CC-RCC correlates with increased serum level of soluble $\mathrm{N}$-terminal E-cadherin fragment. Thus, future analysis of serum E-cadherin of CC-RCC patients may prove to be a valuable biomarker for CC-RCC

Ectodomain shedding of transmembrane proteins is mediated by the action of membrane-associated proteases. The matrix metalloproteases (MMP), cathespins and kallikreins are proteases reported to cleave E-cadherin within its ectodomain. ${ }^{18,21,23,58-62}$ In addition, hepatocyte growth factor/scatter factor (HGF/SF) expression has been associated with E-cadherin ectodomain shedding and increased invasiveness of cancer cells. ${ }^{63}$ The protease(s) responsible for E-cadherin cleavage in CC-RCC has not been identified. However, it has been shown that VHL regulates the expression of tissue inhibitors of metalloproteinases (TIMPs) and MMP2 and 9, and their deregulation have been shown to promote branching morphogenesis in cells devoid of functional VHL. ${ }^{64}$ Furthermore, HGF/SF and its receptor c-MET are frequently overexpressed in CC-RCC. ${ }^{65-68}$ Therefore, it would be of interest to determine whether the overexpression of MMP2, MMP9 or HGF/SF is responsible for the cleavage of E-cadherin in CC-RCC.

Supplementary Information accompanies the paper on the Laboratory Investigation website (http://www.laboratoryinvestigation.org)

\section{ACKNOWLEDGEMENT}

We thank the members of the Ohh laboratory for their helpful discussions and comments. We thank Liz Taylor-Edmonds for VHL sequencing, Dr Loretta MS Lau for her assistance with statistical analysis and Bob Kuba for his assistance in the SCID mouse xenograft assay. This work was supported by funds from the Canadian Cancer Society (CCS)/National Cancer Institute of Canada (NCIC), the Terry Fox Foundation (TFF)/NCIC, the Canadian Institutes of Health Research (CIHR) and the Kidney Foundation of Canada. MLG is a recipient of the TFF/NCIC scholarship. MASJ holds the Farquharson Chair in Kidney Cancer Research at the Princess Margaret Hospital. MO is a Canada Research Chair in Molecular Oncology.

1. Nelson EC, Evans CP, Lara Jr PN. Renal cell carcinoma: current status and emerging therapies. Cancer Treat Rev 2007;33:299-313.

2. Yao $M$, Huang $Y$, Shioi $K$, et al. Expression of adipose differentiationrelated protein: a predictor of cancer-specific survival in clear cell renal carcinoma. Clin Cancer Res 2007;13:152-160.

3. Curti BD. Renal cell carcinoma. JAMA 2004;292:97-100.

4. Escudier B, Eisen T, Stadler WM, et al. Sorafenib in advanced clear-cell renal-cell carcinoma. N Engl J Med 2007;356:125-134.

5. Hudes G, Carducci M, Tomczak P, et al. Temsirolimus, interferon alfa, or both for advanced renal-cell carcinoma. N Engl J Med 2007;356: 2271-2281.

6. Motzer RJ, Hutson TE, Tomczak $\mathrm{P}$, et al. Sunitinib vs interferon alfa in metastatic renal-cell carcinoma. N Engl J Med 2007;356:115-124.

7. Rini $\mathrm{BI}, \mathrm{Campbell} \mathrm{SC}$. The evolving role of surgery for advanced renal cell carcinoma in the era of molecular targeted therapy. J Urol 2007;177:1978-1984.

8. Karakiewicz PI, Briganti A, Chun FK, et al. Multi-institutional validation of a new renal cancer-specific survival nomogram. J Clin Oncol 2007;25:1316-1322

9. Kaelin Jr WG. Molecular basis of the VHL hereditary cancer syndrome. Nat Rev Cancer 2002;2:673-682.
10. Iliopoulos $\mathrm{O}$, Kibel $\mathrm{A}$, Gray $\mathrm{S}$, et al. Tumor suppression by the human von Hippel-Lindau gene product. Nat Med 1995;1:822-826.

11. Gnarra JR, Zhou S, Merrill MJ, et al. Post-transcriptional regulation of vascular endothelial growth factor mRNA by the VHL tumor suppressor gene product. Proc Natl Acad Sci USA 1996;93: 10589-10594.

12. Kondo K, Klco J, Nakamura $\mathrm{E}$, et al. Inhibition of HIF is necessary for tumor suppression by the von Hippel-Lindau protein. Cancer Cell 2002;1:237-246.

13. Kondo K, Kim WY, Lechpammer M, et al. Inhibition of HIF2alpha is sufficient to suppress pVHL-defective tumor growth. PLoS Biol 2003;1:E83.

14. Nelson WJ, Nusse R. Convergence of Wnt, beta-catenin, and cadherin pathways. Science 2004;303:1483-1487.

15. Esteban MA, Tran MG, Harten SK, et al. Regulation of E-cadherin expression by VHL and hypoxia-inducible factor. Cancer Res 2006;66:3567-3575.

16. Evans AJ, Russell RC, Roche O, et al. VHL promotes E2 box-dependent E-cadherin transcription by HIF-mediated regulation of SIP1 and snail. Mol Cell Biol 2007;27:157-169.

17. Krishnamachary $B$, Zagzag $D$, Nagasawa $H$, et al. Hypoxia-inducible factor-1-dependent repression of E-cadherin in von Hippel-Lindau tumor suppressor-null renal cell carcinoma mediated by TCF3, ZFHX1A, and ZFHX1B. Cancer Res 2006;66:2725-2731.

18. Maretzky $T$, Reiss K, Ludwig A, et al. ADAM10 mediates E-cadherin shedding and regulates epithelial cell-cell adhesion, migration, and beta-catenin translocation. Proc Natl Acad Sci USA 2005;102: 9182-9187.

19. Gumbiner BM. Regulation of cadherin adhesive activity. J Cell Biol 2000;148:399-404.

20. Ito K, Okamoto I, Araki N, et al. Calcium influx triggers the sequential proteolysis of extracellular and cytoplasmic domains of E-cadherin, leading to loss of beta-catenin from cell-cell contacts. Oncogene 1999;18:7080-7090.

21. Reiss K, Ludwig A, Saftig P. Breaking up the tie: disintegrin-like metalloproteinases as regulators of cell migration in inflammation and invasion. Pharmacol Ther 2006;111:985-1006.

22. Steinhusen U, Weiske J, Badock V, et al. Cleavage and shedding of E-cadherin after induction of apoptosis. J Biol Chem 2001;276: 4972-4980.

23. Kuefer R, Hofer MD, Gschwend JE, et al. The role of an 80-kDa fragment of E-cadherin in the metastatic progression of prostate cancer. Clin Cancer Res 2003;9:6447-6452.

24. Katayama M, Hirai S, Kamihagi $\mathrm{K}$, et al. Soluble E-cadherin fragments increased in circulation of cancer patients. $\mathrm{Br} J$ Cancer 1994;69:580-585.

25. Lonergan KM, lliopoulos $\mathrm{O}$, Ohh $\mathrm{M}$, et al. Regulation of hypoxiainducible mRNAs by the von Hippel-Lindau tumor suppressor protein requires binding to complexes containing elongins $\mathrm{B} / \mathrm{C}$ and $\mathrm{Cul2}$. Mol Cell Biol 1998;18:732-741.

26. Iliopoulos O, Ohh M, Kaelin Jr WG. pVHL19 is a biologically active product of the von Hippel-Lindau gene arising from internal translation initiation. Proc Natl Acad Sci USA 1998;95: 11661-11666.

27. Ohh M, Yauch RL, Lonergan KM, et al. The von Hippel-Lindau tumor suppressor protein is required for proper assembly of an extracellular fibronectin matrix. Mol Cell 1998;1:959-968.

28. Maynard MA, Evans AJ, Hosomi T, et al. Human HIF-3alpha4 is a dominant-negative regulator of HIF-1 and is downregulated in renal cell carcinoma. FASEB J 2005;19:1396-1406.

29. Ohh M, Park CW, Ivan M, et al. Ubiquitination of HIF requires direct binding to the von Hippel-Lindau protein beta domain. Nat Cell Biol 2000:2:423-427.

30. Feldman $D$, Thulasiraman $V$, Ferreyra $R$, et al. Formation of the VHLelongin $\mathrm{BC}$ tumor suppressor complex is mediated by the chaperonin TRiC. Mol Cell 1999;4:1051-1061.

31. Eble JN, Sauter G, Epstein JI, Sesterhenn I, (eds). World Health Organization Classification of Tumours: Pathology and GeneticsTumours of the Urinary System and Male Genital Organs. IRAC press: Lyon, 2004.

32. Langner $\mathrm{C}$, Ratschek M, Rehak $\mathrm{P}$, et al. Expression of MUC1 (EMA) and E-cadherin in renal cell carcinoma: a systematic immunohistochemical analysis of 188 cases. Mod Pathol 2004;17:180-188. 
33. Blankenship C, Naglich J, Whaley J, et al. Alternate choice of initiation codon produces a biologically active product of the von HippelLindau gene with tumor suppressor activity. Oncogene 1999;18: 1529-1535.

34. Iliopoulos O, Ohh M, Kaelin W. pVHL19 is a biologically active product of the von Hippel-Lindau gene arising from internal translation initiation. Proc Natl Acad Sci USA 1998;95:11661-11666.

35. Schoenfeld A, Davidowitz EJ, Burk RD. A second major native von Hippel-Lindau gene product, initiated from an internal translation start site, functions as a tumor suppressor. Proc Natl Acad Sci USA 1998;95:8817-8822.

36. Stebbins CE, Kaelin WG, Pavletich NP. Structure of the VHL-elonginCelonginB complex: implications for VHL tumor suppressor function. Science 1999;284:455-461.

37. Clifford SC, Cockman ME, Smallwood AC, et al. Contrasting effects on HIF-1alpha regulation by disease-causing pVHL mutations correlate with patterns of tumorigenesis in von Hippel-Lindau disease. Hum Mol Genet 2001;10:1029-1038.

38. Lolkema MP, Gervais ML, Snijckers CM, et al. Tumor suppression by the von Hippel-Lindau protein requires phosphorylation of the acidic domain. J Biol Chem 2005;280:22205-22211.

39. Ohh M, Yauch RL, Lonergan KM, et al. The von Hippel-Lindau tumor suppressor protein is required for proper assembly of an extracellular fibronectin matrix. Mol Cell 1998;1:959-968.

40. Feldman DE, Thulasiraman V, Ferreyra RG, et al. Formation of the VHLelongin $\mathrm{BC}$ tumor suppressor complex is mediated by the chaperonin TRiC. Mol Cell 1999:4:1051-1061.

41. Melville MW, McClellan AJ, Meyer AS, et al. The Hsp70 and TRiC/CCT chaperone systems cooperate in vivo to assemble the von HippelLindau tumor suppressor complex. Mol Cell Biol 2003;23:3141-3151.

42. Hansen WJ, Ohh M, Moslehi J, et al. Diverse effects of mutations in exon II of the von Hippel-Lindau (VHL) tumor suppressor gene on the interaction of pVHL with the cytosolic chaperonin and $\mathrm{pVHL}$ dependent ubiquitin ligase activity. Mol Cell Biol 2002;22:1947-1960.

43. Yao $M$, Yoshida $M$, Kishida $T$, et al. VHL tumor suppressor gene alterations associated with good prognosis in sporadic clear-cell renal carcinoma. J Natl Cancer Inst 2002;94:1569-1575.

44. Han AC, Soler AP, Tang CK, et al. Nuclear localization of E-cadherin expression in Merkel cell carcinoma. Arch Pathol Lab Med 2000;124:1147-1151.

45. Moon KC, Cho SY, Lee HS, et al. Distinct expression patterns of $\mathrm{E}$-cadherin and beta-catenin in signet ring cell carcinoma components of primary pulmonary adenocarcinoma. Arch Pathol Lab Med 2006;130:1320-1325.

46. Serra S, Salahshor S, Fagih M, et al. Nuclear expression of E-cadherin in solid pseudopapillary tumours of the pancreas. J Pancreas 2007;8:296-303.

47. Bindra RS, Vasselli JR, Stearman R, et al. VHL-mediated hypoxia regulation of cyclin D1 in renal carcinoma cells. Cancer Res 2002;62:3014-3019.

48. Russell $\mathrm{RC}$, Ohh $\mathrm{M}$. The role of $\mathrm{VHL}$ in the regulation of E-cadherin: a new connection in an old pathway. Cell Cycle 2007;6:56-59.

49. Marambaud P, Shioi J, Serban G, et al. A presenilin-1/gamma-secretase cleavage releases the $\mathrm{E}$-cadherin intracellular domain and regulates disassembly of adherens junctions. EMBO J 2002;21:1948-1956.
50. Carpenter G. Nuclear localization and possible functions of receptor tyrosine kinases. Curr Opin Cell Biol 2003;15:143-148.

51. Fortini ME. Gamma-secretase-mediated proteolysis in cell-surfacereceptor signalling. Nat Rev Mol Cell Biol 2002;3:673-684.

52. Haas IG, Frank M, Veron $N$, et al. Presenilin-dependent processing and nuclear function of gamma-protocadherins. J Biol Chem 2005;280:9313-9319.

53. Shih leM, Wang TL. Notch signaling, gamma-secretase inhibitors, and cancer therapy. Cancer Res 2007;67:1879-1882.

54. Gofuku J, Shiozaki H, Doki Y, et al. Characterization of soluble E-cadherin as a disease marker in gastric cancer patients. $\mathrm{Br} \mathrm{J}$ Cancer 1998;78:1095-1101.

55. Protheroe AS, Banks RE, Mzimba M, et al. Urinary concentrations of the soluble adhesion molecule E-cadherin and total protein in patients with bladder cancer. Br J Cancer 1999;80:273-278.

56. Ryniers F, Stove C, Goethals $M$, et al. Plasmin produces an E-cadherin fragment that stimulates cancer cell invasion. Biol Chem 2002;383:159-165.

57. Wheelock MJ, Buck CA, Bechtol KB, et al. Soluble 80-kDa fragment of cell-CAM 120/80 disrupts cell-cell adhesion. J Cell Biochem 1987;34:187-202.

58. Gocheva V, Joyce JA. Cysteine cathepsins and the cutting edge of cancer invasion. Cell Cycle 2007;6:60-64.

59. Johnson SK, Ramani VC, Hennings L, et al. Kallikrein 7 enhances pancreatic cancer cell invasion by shedding E-cadherin. Cancer 2007;109:1811-1820.

60. Davies G, Jiang WG, Mason MD. Matrilysin mediates extracellular cleavage of E-cadherin from prostate cancer cells: a key mechanism in hepatocyte growth factor/scatter factor-induced cell-cell dissociation and in vitro invasion. Clin Cancer Res 2001;7:3289-3297.

61. Lee $\mathrm{KH}$, Choi EY, Hyun MS, et al. Association of extracellular cleavage of E-cadherin mediated by MMP-7 with HGF-Induced in vitro invasion in human stomach cancer cells. Eur Surg Res 2007;39:208-215.

62. Symowicz J, Adley BP, Gleason KJ, et al. Engagement of collagenbinding integrins promotes matrix metalloproteinase-9-dependent $\mathrm{E}$ cadherin ectodomain shedding in ovarian carcinoma cells. Cancer Res 2007;67:2030-2039.

63. Kim $\mathrm{CH}$, Kim J, Kahng $\mathrm{H}$, et al. Change of E-cadherin by hepatocyte growth factor and effects on the prognosis of hypopharyngeal carcinoma. Ann Surg Oncol 2007;14:1565-1574.

64. Koochekpour S, Jeffers M, Wang P, et al. The von Hippel-Lindau tumor suppressor gene inhibits hepatocyte growth factor/scatter factorinduced invasion and branching morphogenesis in renal carcinoma cells. Mol Cell Biol 1999;19:5902-5912.

65. Horie S, Aruga S, Kawamata $\mathrm{H}$, et al. Biological role of HGF/MET pathway in renal cell carcinoma. J Urol 1999;161:990-997.

66. Nakaigawa N, Yao M, Baba M, et al. Inactivation of von Hippel-Lindau gene induces constitutive phosphorylation of MET protein in clear cell renal carcinoma. Cancer Res 2006;66:3699-3705.

67. Natali PG, Prat M, Nicotra MR, et al. Overexpression of the met/HGF receptor in renal cell carcinomas. Int J Cancer 1996;69:212-217.

68. Pisters LL, el-Naggar AK, Luo W, et al. C-met proto-oncogene expression in benign and malignant human renal tissues. J Urol $1997 ; 158: 724-728$ 